\title{
ZIGBEE BASED-DIFFERENTIAL PILOT PROTECTION OF TRANSMISSION LINE
}

\author{
Jyoti Thakur ${ }^{1}$, Hemant Pant ${ }^{2}$ \\ ${ }^{I} M-T e c h$, Instrumentation, Punjab University, Chandigarh, India \\ ${ }^{2} M-T e c h$, Instrumentation, Punjab University, Chandigarh, India
}

\begin{abstract}
This paper describes the limitations of the existing relays in the detection of fault. It also describes the benefits of using the differential pilot protection of the transmission line. Among other advantages one of its main advantages is its speed, which is required in a.c. systems to remain in synchronism (as angle $\delta$ is to be kept small). In the end of this paper a model has been created in the laboratory by using two incandescent bulbs in parallel to create the difference in the two parts of the model, this difference is detected and appropriate action is finally taken to trip the circuit.
\end{abstract}

Keywords: Zig-bee, pilot protection, differential protection, transmission line, Hall Effect sensors. $* * *$

\section{WHY DO WE NEED PROTECTION IN} POWER SYSTEM?

There are many reasons for the protection in the power system such as:-

- $\quad$ To prevent Apparatus like:-

1. Transmission Line Protection

2. Transformer Protection

3. Generator Protection

4. Motor Protection

5. Busbar Protection

- To prevent personnel's.

- To remain in synchronism (reliability of power).

It has been seen that most faults occurs in overhead transmission line, therefore it becomes mandatory to protect the transmission line.

\section{LIMITATIONS IN EXISTING METHODS}

\section{Cannot Differentiate between Inrush Currents and}

\section{Fault Currents}

All the Existing Relays cannot Differentiate between the fault currents and the inrush currents, for example in case of the transformer switching, the inrush current is equal to that of the fault current so extra relay has to be used that is , the harmonic restrained relay. The harmonic restrained relay can identify the transformer inrush current and will not trip as it contains harmonics whereas the normal relays like mho or impedance alone would not have been able to do this all alone.

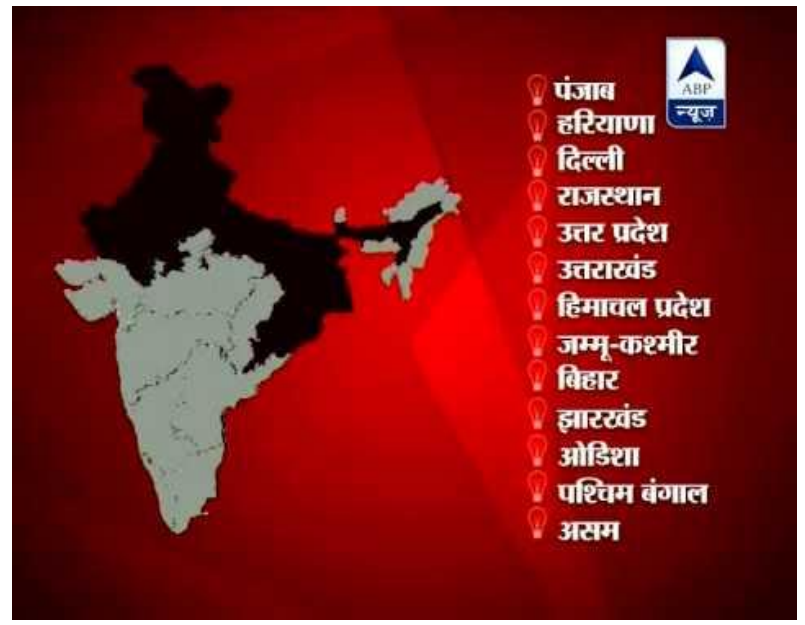

Fig -1 Blackout in India

One of the most severe blackout in India happened on $30^{\text {th }}$ and $31^{\text {st }}$ July 2012 . The complete failure of the northern and north eastern grid effected the life or more than 3 million people. The three states Uttar Pradesh, Punjab and Haryana were drawing more power than that was expected from them. Which made the load current equal to the fault current. The relays were not able to differentiate between the load current and the fault current due to which $400 \mathrm{kV}$ Bina-Gwalior line tripped leading to the cascading effect.

\section{No Precisely Defined Boundaries}

The present relays do not have precisely defined boundaries. The transmission lines are divided into parts called zones. Each zone is given two protections: primary protection and back up protection. As shown in figure-2 due to the imprecisely defined boundaries the part of the transmission line can remain unprotected which is known as dead zone. 


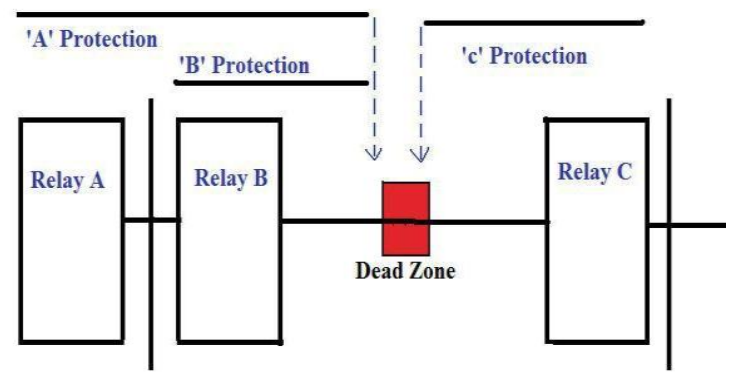

Fig -2 Dead Zone in Transmission line

Although a safety factor is always considered and this problem is very rare.

\section{Magnitude as Well as Direction}

The decision of tripping the transmission lines can not only be taken on the basis of magnitude of the fault current. Relays like Mho can sense magnitude as well as direction of current whereas the reactance relay cannot sense the direction. So most of the times, these relays have to be used in pairs to achieve the directional characteristics.

\section{DIFFERENTIAL PILOT PROTECTION.}

Before we move further let us see how a percentage differential protection in transformers is applied.

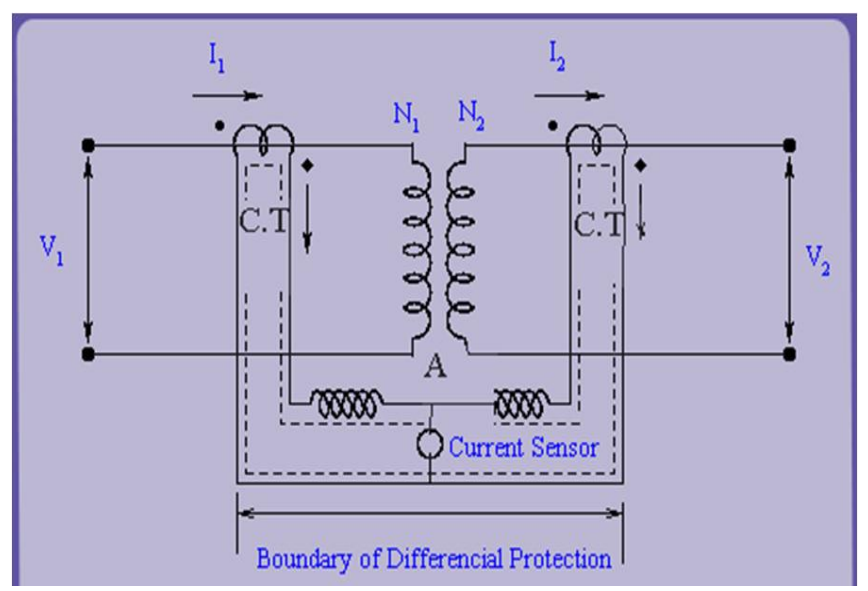

Fig -3 Differential Protection of Transformers

During normal conditions the induced currents in the arms of CT are equal (considering by proper adjustment of the turn ratio) and therefore no current flows through the current sensors. But with the occurrence of fault (within the boundaries of CT's) there is different induced currents in the CT's and therefore there is current in the current sensor. Hence tripping signal is sent to the Circuit Breaker.

From the above said scheme it becomes quite obvious that this scheme cannot be implemented in the transmission lines as it is. Firstly, because of the extra cost that needs to be invested in the pilot wires, and finally the high maintenance cost that will go in the protecting system itself. Therefore this scheme needs to be modified first before it can be implemented in the transmission lines.

\subsection{Using differential Pilot Protection in}

\section{Transmission Line}

To use differential protection in transmission lines the pilot wires are replaced by the communicating equipment and here difference of two current readings is taken by using micro-controller. Instead of using restraining coil the adjustments are done in the algorithms of micro-controller.

By doing so the concepts of differential protection can also be implemented in the transmission line which will lead to the following advantages:-

- $\quad$ Precisely defined boundaries.

- Magnitude as well as direction can be sensed.

- Fast Tripping time is achieved as the data from different parts of transmission line is available.

Instead of using the relays as the sensing part if it can be replaced by other current sensors such as Hall Effect current sensors thus the tripping time can be further decreased leading to more system stability in terms of synchronism. As Hall Effect sensors have less response time when compared with conventional relays.

- $\quad$ The errors due to charging currents in medium and long transmission line can be eliminated by the use of proper algorithm.

\section{LITERATURE SURVEY}

The work done by the working group $8,[1]$ has given many advantages of using the differential pilot protection for the transmission lines, one of the basic requirement for the stability of transmission line is the speed of the tripping as governed by the equal area criteria and differential pilot protection is one of the scheme that promises the speedy operation but the reason of not implementing this scheme is the cost of the communication equipment. Whereas Mustahsan Mir and Patrick J McCleer[2] has shown that by taking 12 samples per cycle and using the pre-fault and postfault data the internal and external faults can be detected. The delta currents are in different quadrant if external fault occurs whereas the delta currents are in same quadrant if the occurrence of fault is internal. The calculation of charging current has also been done. By modeling the short transmission line in the lab (using lumped parameters) they have proved the advantages of differential pilot protection, although no wireless equipment was used. Whereas M. G. Adamiak and W. Premerlani [3] has discussed the solutions of increasing the speed of differential pilot protection of transmission lines by using the Fourier Transform, the issues of increasing the reliability in case of loss of channel of communicating equipment has also been discussed. The techniques such as GPS and Ping Pong have also been discussed to synchronize the data samples. They have also shown that only those packets with same time tag be compared. While Armando Guzma'n, Joe Mooney, Gabriel Benmouyal and Normann Fischer [4] has discussed that if many different length data-window will be used the reliability as well as the speed can be increased considerably. P. Chiradeja and A. Ngaopitakkul [5] have used artificial neural networks and discrete wavelet 
transform to differentiate the faults. B. Ravindhranath Reddy[6] has also used wavelet transform to detect the fault locations. Abdel-Latif, K.M.[7] and his team mates has shown how we can use a Wi-Fi protocol as a communication source in the protection of transmission line.

\section{PREPARING THE MODEL}

The response time of the relay is usually more than as compared to the Hall Effect sensors. So faster operation can be achieved if the relays can be replaced by the Hall Effect sensors. Another problem that is being faced in the implementation of the differential pilot protection of the transmission line is the cost of the communicating equipment. So here we can use Zigbee chips instead of the costly equipments. The maximum range of the Zig-bee is around $2 \mathrm{kms}$, and more than 65000 Zig-bee's (theoretically) can be connected in a network. Thereby the effective protective distance can be increased. The following figure shows how a network can be implemented to increase the distance and how the packet can be delivered with the minimum time.

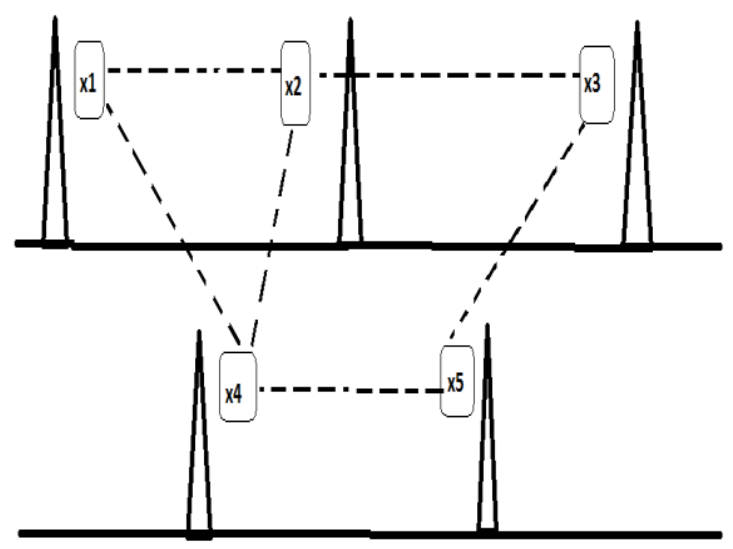

Fig -4 Mesh topology in transmission line

Here the $\mathrm{X}$-bee chips $\mathrm{x} 4$ is not only connected to $\mathrm{x} 5$ i.e. one circuit of transmission line but also to other X-bee's i.e. $\mathrm{x} 1$ and $\mathrm{x} 2$ of another circuit (provided they are in range). This network is connected in mesh topology, and therefore it can be easily discerned that the range can be increased and if the $\mathrm{x} 2$ goes down the data from $\mathrm{x} 1$ to $\mathrm{x} 3$ can be sent through $\mathrm{x} 4$. No external programming has to be done to set the protocol as Zig-Bee protocol itself supports the mesh topology and itself finds the shortest path to the destination.

\section{Hardware}

To create the difference between the currents at different parts of the circuit, a model has been created using two incandescent bulbs of 220 Volts, 100 Watts each. The resistance of each bulb comes out to be $484 \mathrm{Ohms}$. When the bulb (acting as a load) is switched ON, the current at point $A$ and point $B$ is same and both Hall effect sensors reads the same value. But if the second bulb is also switched $\mathrm{ON}$ (acting as a fault), the current at point B is more than the current at point $\mathrm{A}$.

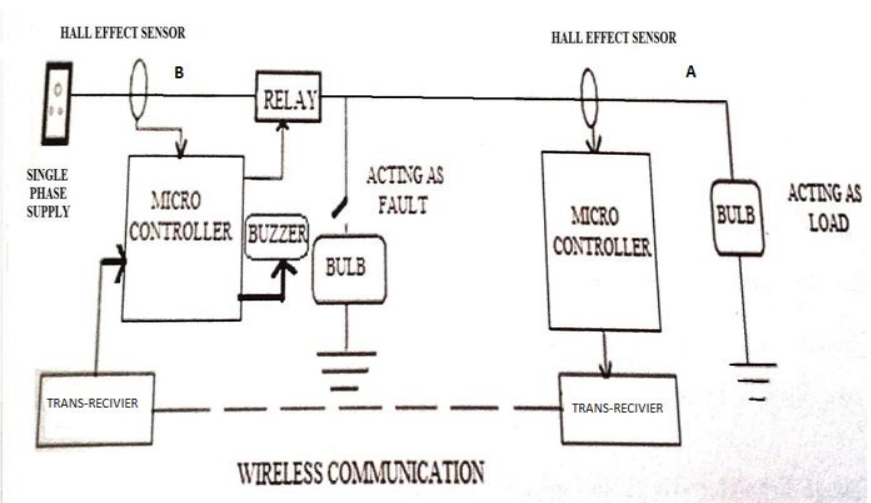

Fig -5 Block Diagram of the Model

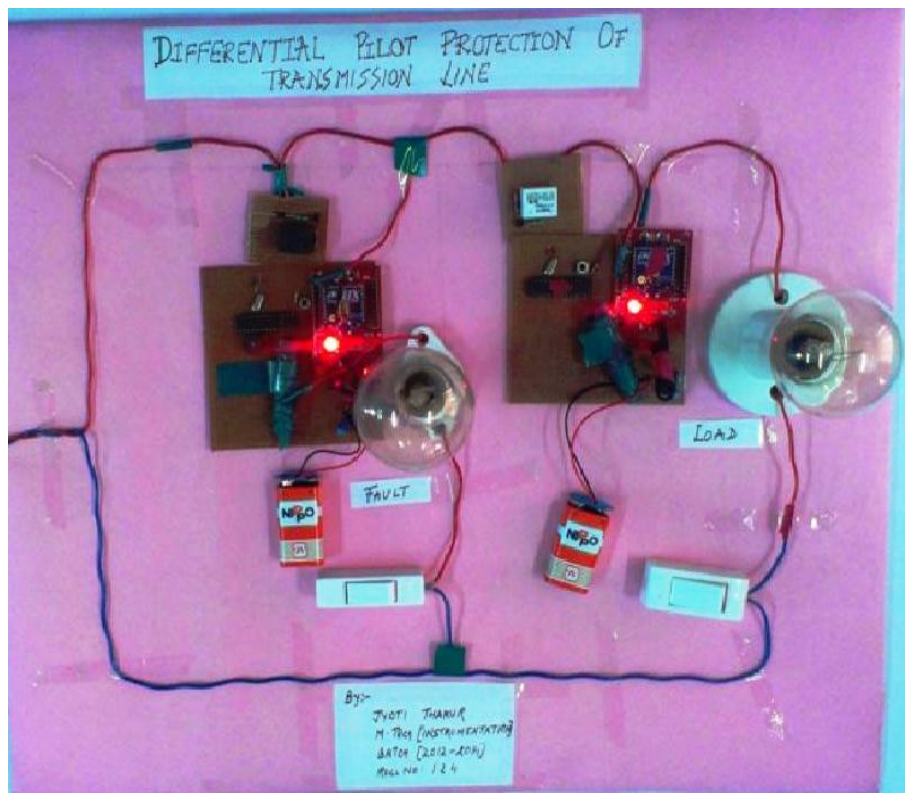

Fig -6 Physical Model

At different situations the response of the circuit can be summarized as shown in the Table 1.

Table -1

\begin{tabular}{|l|l|l|l|}
\hline & $\begin{array}{l}\text { CURRENT } \\
\text { AT POINT } \\
\text { A }\end{array}$ & $\begin{array}{l}\text { CURRENT } \\
\text { AT POINT } \\
\text { B }\end{array}$ & $\begin{array}{l}\text { DECISION } \\
\text { BY Micro- } \\
\text { Controller } \\
\text { At A }\end{array}$ \\
\hline $\begin{array}{l}\text { When Load } \\
\text { Bulb is ON }\end{array}$ & 0.45 Amps & 0.45 Amps & $\begin{array}{l}\text { No Tripping } \\
\text { Signal }\end{array}$ \\
\hline $\begin{array}{l}\text { When Both } \\
\text { Bulbs are } \\
\text { ON }\end{array}$ & 0.45 Amps & 0.9 Amps & $\begin{array}{l}\text { Line is } \\
\text { Tripped }\end{array}$ \\
\hline
\end{tabular}

It can be clearly seen from the table that when both the bulbs are switched $\mathrm{ON}$ there is a difference between the currents at point $\mathrm{A}$ and $\mathrm{B}$ and hence, tripping action is taken. The algorithms of the microcontrollers of both sides is shown in figure 6. 

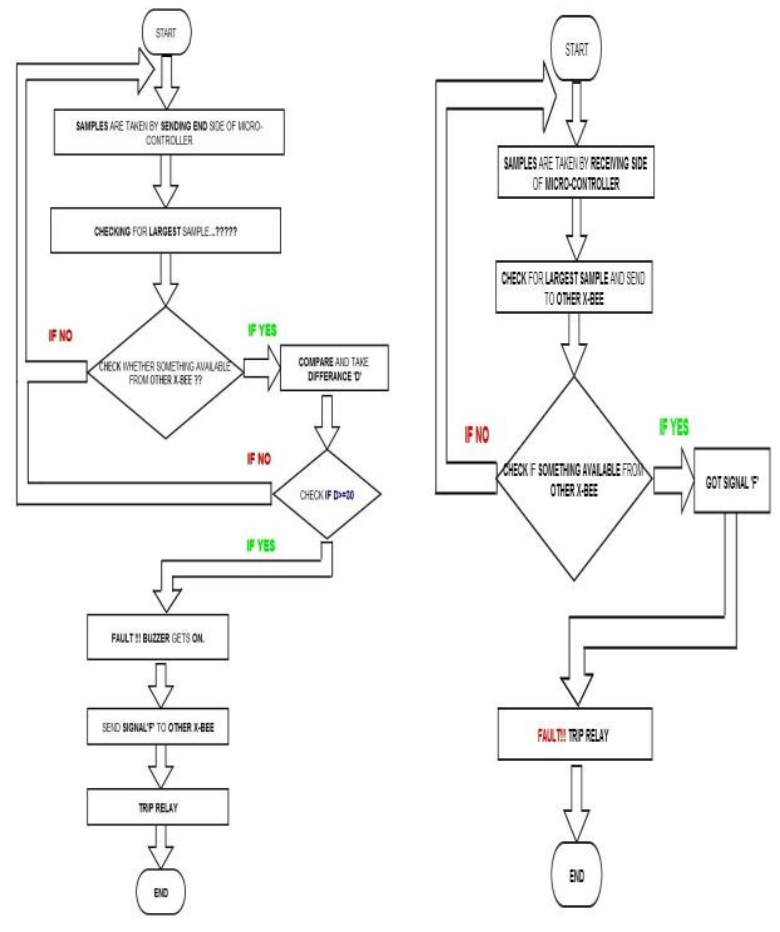

Fig -6 Algorithms of two Microcontroller's, of each side

Twenty four samples are taken in every cycle of a.c. wave $(20 \mathrm{msec})$. The highest sample is calculated and transferred to the sending side micro-controller. The sending side micro-controller compares the difference between the two values(highest samples) and take the appropriate action.

Although more sophisticated methods can be created, to decrease the probability of mal-operation due to the missing samples the Fourier transform window method or phaselets method could be used. The second thing which also must be considered here is that the samples of same instant must be compared which makes it necessary to use the concept of time tag.

\section{CONCLUSION}

Using differential pilot protection for the protection of transmission line has many advantages as already stated. But one of the reasons of not its implication is the cost of communicating equipment, which could be overcome by using Zig-bee pro chips which has a descent range of $2 \mathrm{Kms}$. Zig-bee chips can be connected in mesh topology and therefore the range can be further increased if they are smartly placed.

\section{ACKNOWLEDGEMENTS}

We are thankful to Punjab University, UCIM department for providing us the lab and necessary equipments for our experiments and our guides Assistant Professor Mr. H.P.S Kang and Assistant Professor Mr. Anil Kumar for their support and guidance.

\section{REFERENCES}

[1]. "JUSTIFYING PILOT PROTECTION ON TRANSMISSION LINES", A report to the Line Protection Subcommittee D Power System Relaying Committee IEEE Power Engineering Society Prepared by Working Group D8. [2]. "A NEW MICROCOMPUTER-BASED APPROACH FOR PILOT DIFFERENTIAL PROTECTION OF TRANSMISSION LINES", Mustahasan Mir and Patrick J McCleer, Umm Al-Qura University Saudi Arabia and University of Michigan, Ann Arbor, MI ,U.S.A.

[3]. "A New Approach to Current Differential Protection for Transmission Lines", by M. G. Adamiak et al.

[4]. "Transmission line protection system for increasing power system requirements", Guzman,A., Schweitzer Eng. et al.

[5]. Identification of Fault Types for Single Circuit Transmission Line using Discrete Wavelet transform and Artificial Neural Networks P. Chiradeja and A. Ngaopitakkul, Member, IAENG

[6]. "Fault detection, classification and location on transmission lines using wavelet transform", B. Ravindhranath Reddy et al.

[7]. " Laboratory Investigation of Using Wi-Fi Protocol for Transmission Line Differential Protection", Abdel-Latif et al.

\section{BIOGRAPHIES}

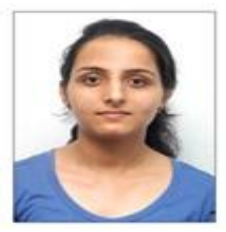

Miss. Jyoti Thakur is B-Tech in Electrical Engineering and M-Tech in Instrumentation from Punjab University, Chandigarh. Her research interests include wireless technology.PLC and protection of Power Systems.

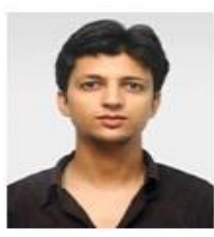

Mr. Hemant Pant is B-Tech in Electrical Engineering and M-Tech in Instrumentation from Punjab University, Chandigarh. The author has keen interests in microcontrollers, PLC, wireless technology and embedded systems. 